\title{
Design of Broadband Quasi-Yagi Antenna Using a Folded Dipole Driver
}

\author{
Son Xuat $\mathrm{Ta}^{1} \cdot$ Byoungchul $\mathrm{Kim}^{1} \cdot \mathrm{Hosung}_{\mathrm{Choo}^{2}} \cdot \mathrm{Ikmo} \mathrm{Park}^{1}$
}

\begin{abstract}
This paper describes the development of a broadband quasi-Yagi antenna using a folded dipole driver. The antenna is designed on a low-permittivity substrate to reduce the surface wave effect, and hence the gain can be enhanced easily by adding directors. The folded dipole driver is connected to a $50-\Omega$ microstripline via a simple broadband microstrip-to-coplanar stripline transition with a quarter radial stub. The key motivation for the use of a folded dipole is to increase the input impedance at the driver, allowing a smaller mismatch loss between the antenna driver and the coplanar stripline feed. The proposed antenna has a measured bandwidth of $4.67 \sim 6.26 \mathrm{GHz}$ for the $-10 \mathrm{~dB}$ reflection coefficient, and a flat gain of $4.86 \sim 5.15 \mathrm{~dB}$ within the bandwidth.
\end{abstract}

Key words: Broadband, Quasi-Yagi Antenna, Folded-Dipole.

\section{Introduction}

In recent years, quasi-Yagi antennas have been widely employed in microwave and millimeter-wave applications, due to their broad bandwidth, high efficiency, high directivity, low profile, uniplanar structure, ease of fabrication, and low cost. These antennas can be designed on a high-permittivity substrate [1] [8] or a lowpermittivity substrate [9] [13] with a regular-dipoledriver feed and various types of feedlines, including microstriplines [1], [2], [9 13], coplanar waveguides [3] [6], coplanar striplines [7], or slotlines [8]. If the antenna is designed on a high-permittivity substrate, the gain cannot be enhanced easily by adding directors, due to the surface wave effect. This effect can be reduced by using a low-permittivity substrate. However, when the antenna is designed on a low-permittivity substrate, a regular dipole driver is not quite compatible with a coplanar stripline feed, since the characteristic impedance of such a feedline is usually much higher than the input impedance of a regular dipole. Recently, folded dipole driven quasi-Yagi antennas [14], [15] have been reported with a microstrip-to-coplanar stripline transition feed which introduced a $180^{\circ}$ phase delay between two microstripline branches by adjusting their length. However, this transition is guaranteed only at the center frequency of operation.

This paper introduces a broadband folded-dipole-driven quasi-Yagi antenna that is built on a low-permittivity substrate. The folded dipole, which is utilized to increa- se the input impedance at the driver, is fed by a coplanar stripline that is matched with a $50-\Omega$ microstripline by a quarter radial stub [14]. The antenna utilizes a parasitic strip as a director and a truncated ground plane as the reflector in order to minimize the gain variation.

\section{Antenna Design and Characteristics}

Fig. 1 shows the geometry of the folded-dipole-driven quasi-Yagi antenna. The antenna was designed on both sides of a $60 \times 60 \mathrm{~mm}$ Rogers RO4003 substrate with a relative dielectric constant of 3.38 and a thickness of $0.508 \mathrm{~mm}$. The antenna is comprised of a microstrip feedline, a folded dipole driver, a strip parasitic element used as a director, and the truncated ground plane used as a reflector. The ground plane was placed on the back side of the substrate, with a length of $30 \mathrm{~mm}$ and a width of $60 \mathrm{~mm}$. The driver and the director were printed on the top side of the substrate, with a driver-to- director spacing of $S$. The initial length of the driver was approximately half the effective wavelength $\left(\lambda_{\text {eff }} / 2\right)$ at 5 GHz. The director was designed to achieve an optimal phasing for a small gain variation at $5 \sim 6 \mathrm{GHz}$ with the length and width of $L_{d i r}=13 \mathrm{~mm}$ and $W_{c}=3 \mathrm{~mm}$, respectively. The feedline was designed to have a characteristic impedance of $50 \Omega$ for a microstripline width of $W_{m s}=1.14 \mathrm{~mm}$. Since the structure of the antenna prevents the microstrip feedline from being directly connected to the driver, a broadband microstrip-to-coplanar stripline with a radial stub (of radius $R$ and angle $a$ ) was

\footnotetext{
Manuscript received May 27, 2011 ; revised August 24, 2011. (ID No. 20110527-02J)

${ }^{1}$ School of Electrical and Computer Engineering, Ajou University, Suwon, Korea.

${ }^{2}$ School of Electronic and Electrical Engineering, Hongik University, Seoul, Korea.

Corresponding Author : Ikmo Park (e-mail : ipark@ajou.ac.kr)
} 


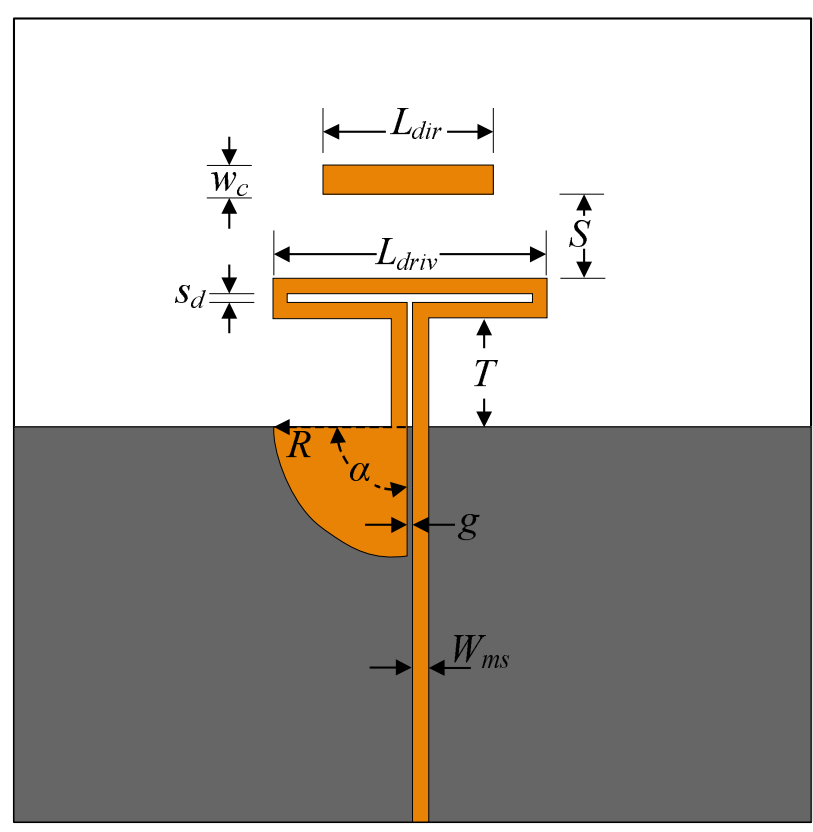

Fig. 1. Folded dipole quasi-Yagi antenna geometry: $W_{m s}=$ $1.14 \mathrm{~mm}, \alpha=90^{\circ}, g=0.5 \mathrm{~mm}, R=10 \mathrm{~mm}, T=8 \mathrm{~mm}$, $L_{d r i v}=21 \mathrm{~mm}, L_{d i r}=13 \mathrm{~mm}, s_{d}=0.6 \mathrm{~mm}, S=5 \mathrm{~mm}$, and $w_{c}=3 \mathrm{~mm}$.

used to make the transition between the two. The coplanar stripline was designed with a characteristic impedance of $146 \Omega$; its length and gap size are $T$ and $g$, respectively. $R, a$, and $T$ were optimised for the wideband impedance matching condition.

The variation of the antenna characteristics as functions of the main design parameters was investigated via the full-wave electromagnetic simulator MWS (by CST). Fig. 2 shows the simulated input impedances of folded and regular dipoles. Two identical dipoles (length of 21 $\mathrm{mm}$, width of $1.14 \mathrm{~mm}$, and feedgap of $0.5 \mathrm{~mm}$ ) were placed on the top side of the substrate without the ground plane, transition, and coplanar stripline feed. In the simulations, the dipoles were excited by discrete port and Z-parameters were computed. As shown in Fig. 2, at $5 \mathrm{GHz}$, these were approximately $150 \Omega$ and $40 \Omega$, respectively. Thus, the folded dipole was chosen for connection with the coplanar stripline, which has a characteristic impedance of approximately $146 \Omega$. This allows a smaller mismatch loss between the antenna driver and the coplanar stripline feed.

The folded-dipole-driven quasi-Yagi antenna is fed by a simple microstrip-to-coplanar stripline transition with a radial stub, designed to obtain the most appropriate impedance matching conditions. These can be inferred from Fig. 3, which shows the simulated reflection coefficient with respect to radius $(R)$ and angle $(\alpha)$ of the radial stub. Fig. 3(a) clearly shows that changing the radius of

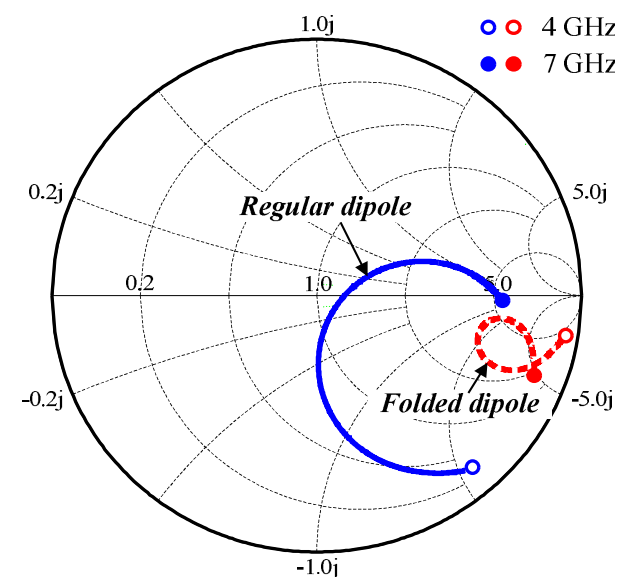

Fig. 2. Simulated input impedances of the regular- and folded-dipoles with the same length of $21 \mathrm{~mm}$ on Smith chart from 4 to $7 \mathrm{GHz}$.

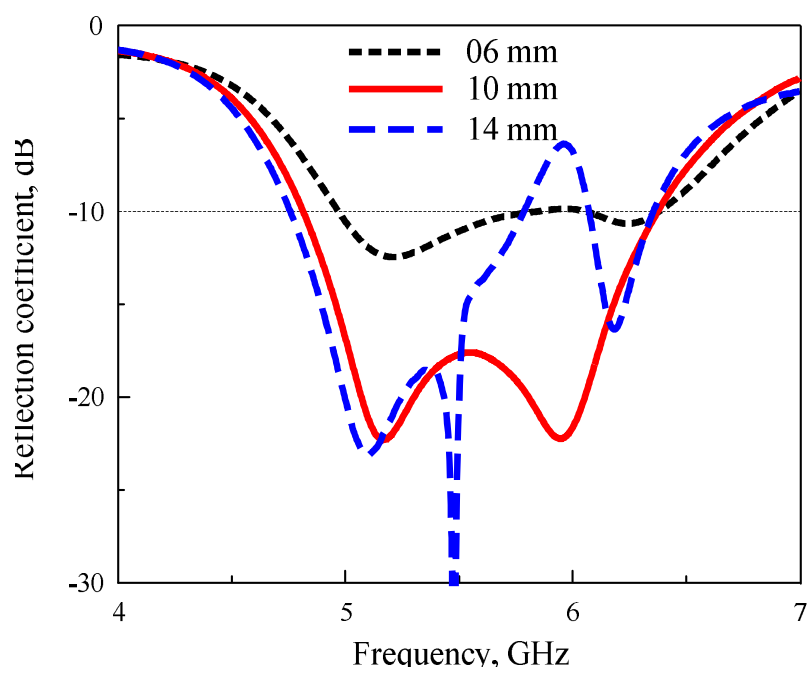

(a) Radius $(R)$

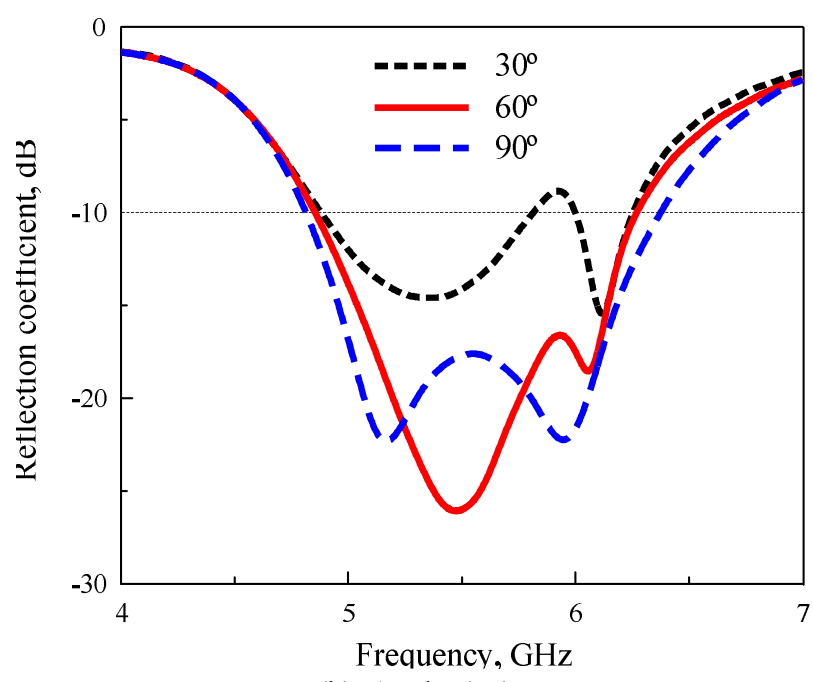

(b) Angle $(\alpha)$

Fig. 3. Reflection coefficient as a function of the radial quarter stub. 
the stub significantly influences the reflection coefficient, and a $10-\mathrm{mm}$ radius offers the best match.

According to Fig. 3(b), as the stub angle increases, the reflection coefficient improves. These two plots indicate that the reflection coefficient is optimized by using a quarter radial stub $\left(\alpha=90^{\circ}\right)$ with a radius of 10 $\mathrm{mm}$. Since the effect of the radial stub on the radiation pattern of the antenna is negligible, it is not shown here.

The coplanar stripline is used as the feed for the folded dipole driver. The length of the coplanar stripline is also equal to the space between the reflector (ground plane) and the driver. The reflection coefficient and the gain of the antenna were simulated for varying coplanar stripline length, and the results are shown in Fig. 4. The effects of the coplanar stripline length $(T)$ on the antenna

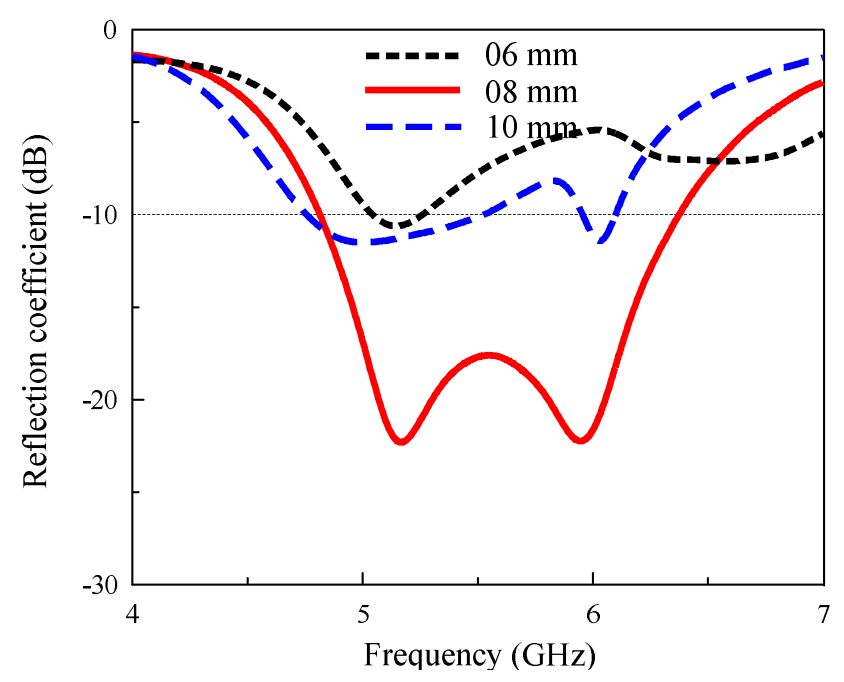

(a) Reflection coefficient

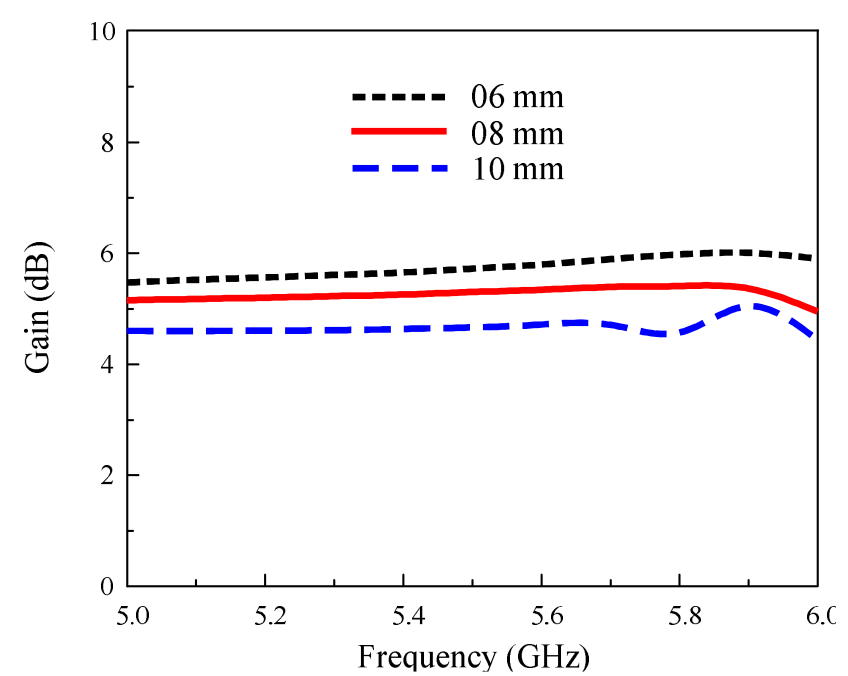

(b) Gain

Fig. 4. Antenna characteristics as functions of the coplanar stripline length $(T)$. characteristics are relatively significant. The reflection coefficient is optimized at a length of $8 \mathrm{~mm}$, which is approximately one-quarter of the effective wavelength $\left(\lambda_{\text {eff }} / 4\right)$ at the center frequency of $5.5 \mathrm{GHz}$ (see Fig. 4(a)). As the coplanar stripline length was increased from $6 \mathrm{~mm}$ to $10 \mathrm{~mm}$ (in increments of $2 \mathrm{~mm}$ ), the gain also increased, as shown in Fig. 4(b). The best gain flatness was observed at a coplanar stripline length of $8 \mathrm{~mm}$.

Fig. 5 shows the simulated reflection coefficient of the folded-dipole-driven quasi-Yagi antenna with respect to driver length. As the length was increased from $20 \mathrm{~mm}$ to $22 \mathrm{~mm}$ (in increments of $1 \mathrm{~mm}$ ), the center frequency decreased, but the bandwidth for the $-10 \mathrm{~dB}$ reflection coefficient did not change significantly. This indicates that the center frequency of the operating bandwidth is mainly determined by the length of the folded-dipole driver. However, the effect of the driver length on the radiation pattern of the antenna is negligible.

Fig. 6 shows the variations of the antenna characteristics as functions of space $S$ between the driver and the director. As the space varied from $2 \mathrm{~mm}$ to $8 \mathrm{~mm}$ (in steps of $3 \mathrm{~mm}$ ), the reflection coefficient changed slightly in the high-frequency region, but was unchanged in the low-frequency region (see Fig. 6(a)), while the antenna gain increased throughout the operating bandwidth (see Fig. 6(b)).

Because of the surface wave effect, the gain of a quasi-Yagi antenna is not as readily increased by adding directors, compared to the case of a conventional YagiUda antenna. To avoid this problem, the proposed antenna was designed on a low-permittivity substrate. The increased gain effect is then evident in Fig. 7, which

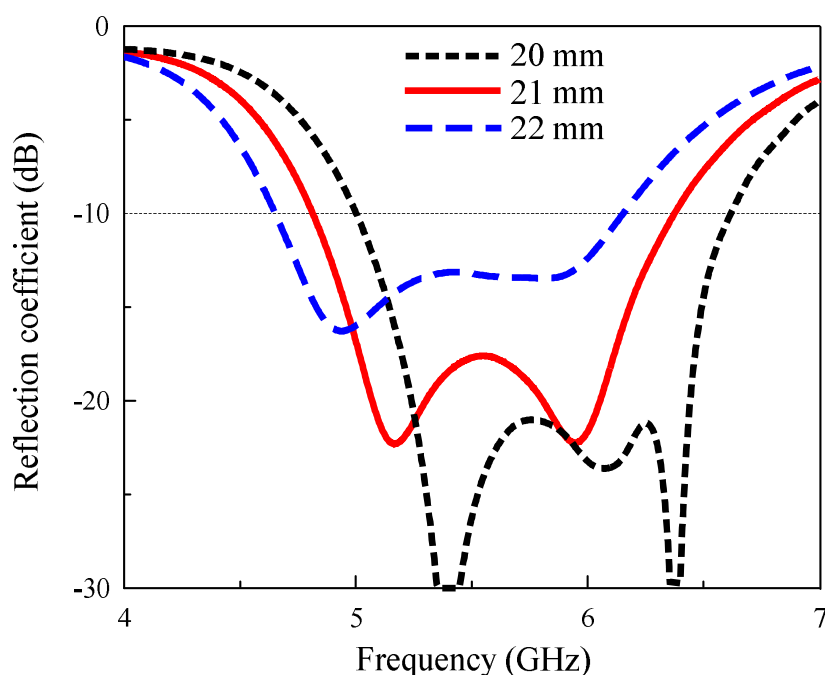

Fig. 5. Reflection coefficient as a function of the driver length $\left(L_{d r i v}\right)$. 


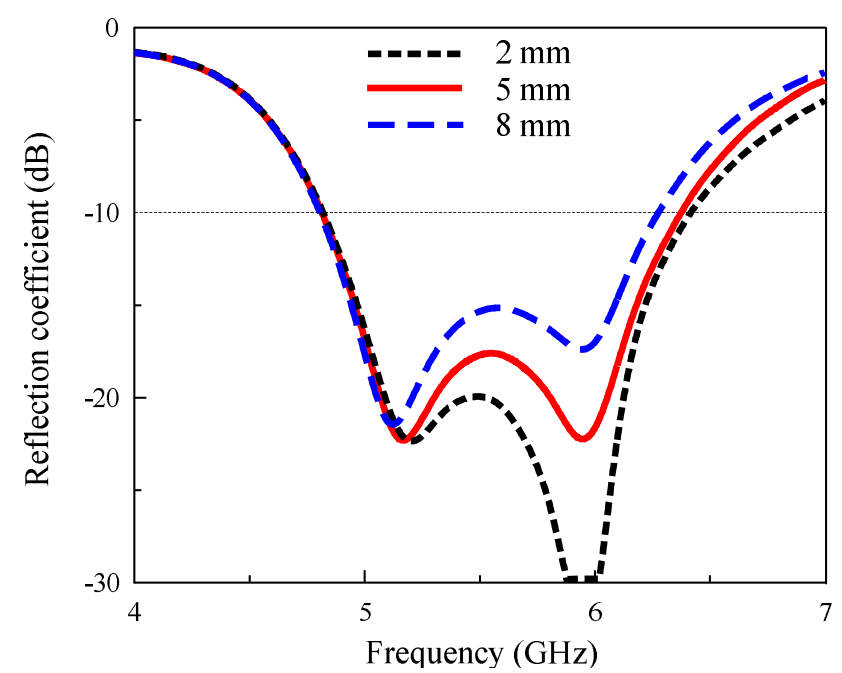

(a) Reflection coefficient

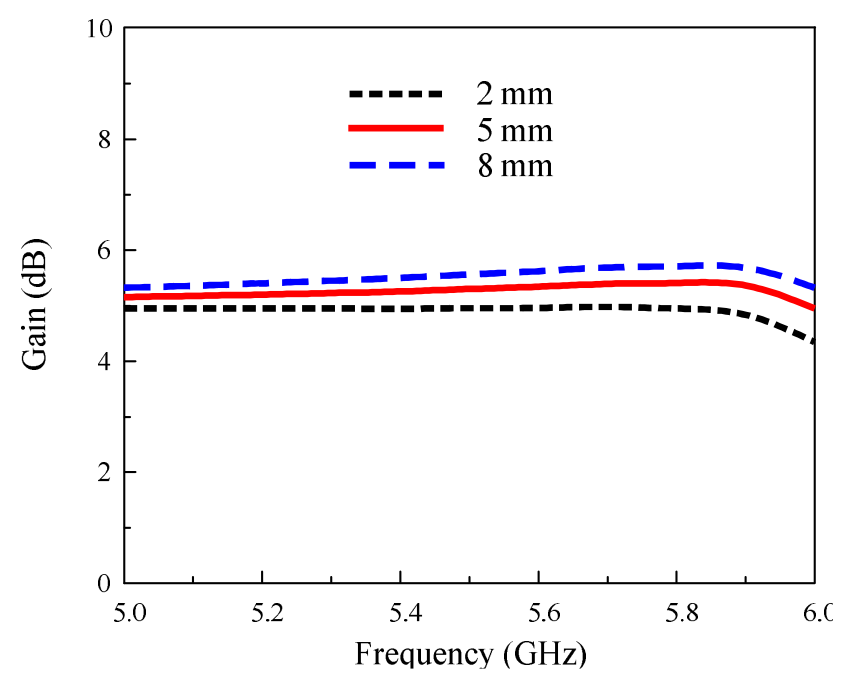

(b) Gain

Fig. 6. Antenna characteristics as functions of the space between the folded-dipole-driver and the director $(S)$.

shows the gain as a function of the number of directors. As the figure indicates, the simulated antenna gains were $4.95 \sim 5.41,6.67 \sim 7.45$, and $7.8 \sim 8.9 \mathrm{~dB}$ at $5 \sim 6$ $\mathrm{GHz}$ for 1-, 5- and 10-director designs, respectively, with a driver-to-director spacing of $5 \mathrm{~mm}$. This indicates that the gain of the proposed antenna is linearly proportional to the number of directors. However, the final antenna design uses only one director with a moderate gain of $4.95 \sim 5.41 \mathrm{~dB}$, since the smallest gain variation is obtained with this configuration. The 5- and 10-director designs have a larger gain variation than the single director design owing to a non-optimal phasing condition of the director elements.

The antenna was fabricated on a Rogers RO4003 substrate with a copper thickness of $20 \mu \mathrm{m}$ via a standard etching technique, and an SMA connector was used for

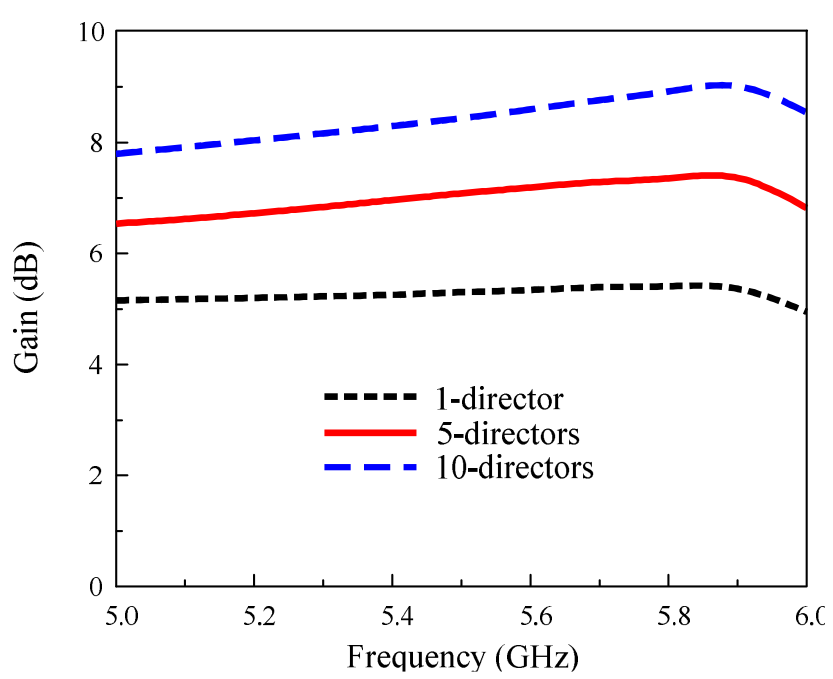

Fig. 7. Gain variation as a function of the number of directors.

the microstrip-to-coaxial line transition (not included in the simulation). An Agilent N5230A network analyzer

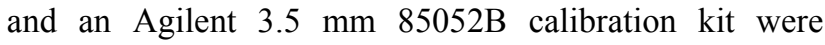
used for measurements. Fig. 8(b) shows good agreement between the simulated and measured reflection coefficients of the optimized antenna. The measured bandwidth for the $-10 \mathrm{~dB}$ reflection coefficient was $4.67 \sim 6.26$ $\mathrm{GHz}$, while the simulated bandwidth was $4.8 \sim 6.3 \mathrm{GHz}$. There were some minor discrepancies between the simulation and the measurements, due to the effect of the coaxial-to-microstripline transition (SMA connector).

The radiation patterns and gain of the broadband folded-dipole-driven quasi-Yagi antenna were measured with an Agilent Vector Network Analyzer (E8362B). Two identical standard horn antennas (one for transmitting and one for receiving) were first connected to two ports of the network analyzer for calibration. The distance between the transmitting and receiving antennas was $10 \mathrm{~m}$. The receiving antenna was then replaced with the proposed antenna. In the measurement process, the transmitting antenna was fixed, while the receiving antenna was rotated from $-180^{\circ}$ to $180^{\circ}$, with a scanning angle of $1^{\circ}$. The 5.0, 5.5, and $6.0 \mathrm{GHz}$ radiation patterns of the antenna in the $E$ - and $H$-planes are illustrated in Fig. 9. Again, the simulated and measured results agreed quite closely, with a front-to-back ratio better than 14, and a cross- polarization level better than $-15 \mathrm{~dB}$. The radiation patterns were not quite symmetrical, since the transition feed was asymmetric. At a frequency of 5 $\mathrm{GHz}$, the half-power beamwidth (HPBW) obtained from the measurements was $76^{\circ}$ along the $E$-plane and $191^{\circ}$ along the $H$-plane. At the center frequency of $5.5 \mathrm{GHz}$, the HPBW obtained from the measurements was $84^{\circ}$ 


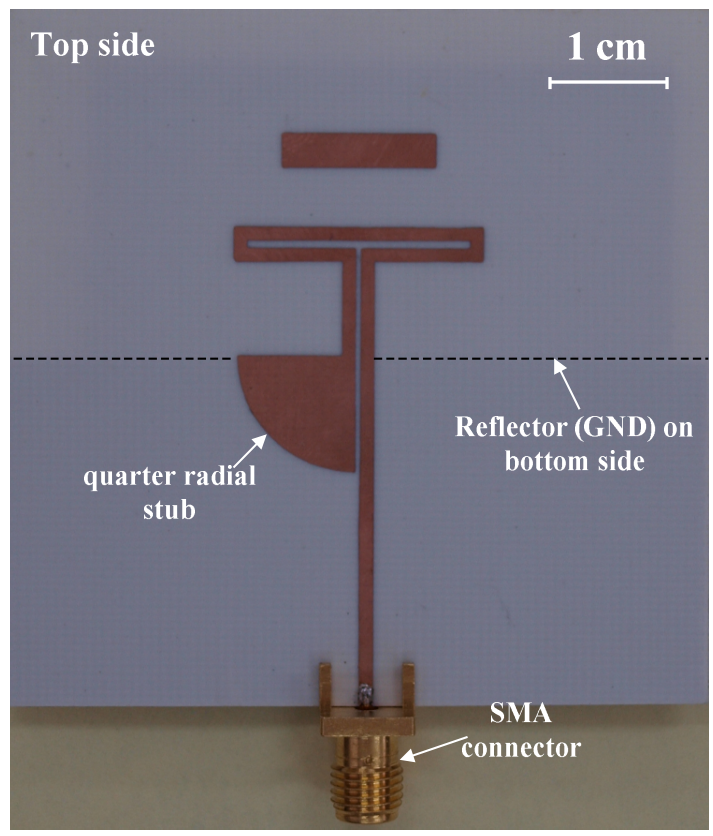

(a)

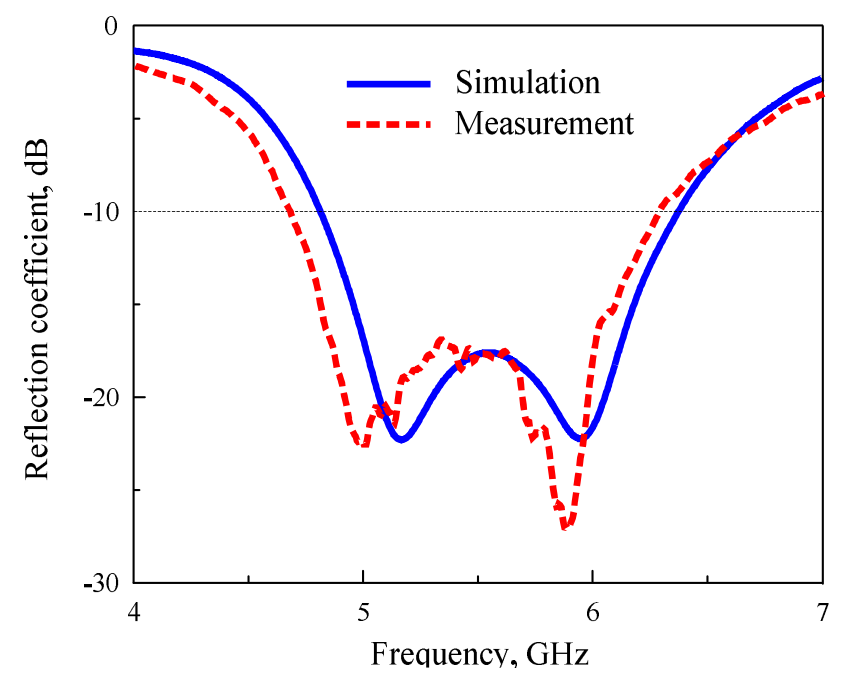

(b)

Fig. 8. (a) Fabricated folded-dipole-driven quasi-Yagi antenna, (b) measured and simulated reflection coefficient.

along the $E$-plane and $187^{\circ}$ along the $H$-plane. At 6 $\mathrm{GHz}$, the HPBW obtained from the measurements was $86^{\circ}$ along the $E$-plane and $182^{\circ}$ along the $H$-plane. As Fig. 10 shows, the measured gain of the antenna was $4.86 \sim 5.15 \mathrm{~dB}$ over the $5 \sim 6 \mathrm{GHz}$ band, and this is in close agreement with the simulated gain of $4.95 \sim 5.41$ $\mathrm{dB}$. The antenna also exhibited a very small gain variation across its bandwidth: the measured gain variation was $0.29 \mathrm{~dB}$ and the simulated gain variation was 0.46 $\mathrm{dB}$. Therefore, this antenna can be stably operated in transmitting or receiving applications in a wireless communication system.

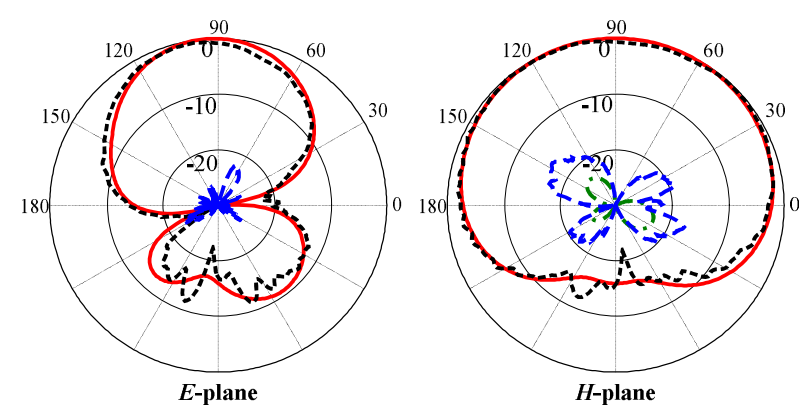

(a) $5 \mathrm{GHz}$

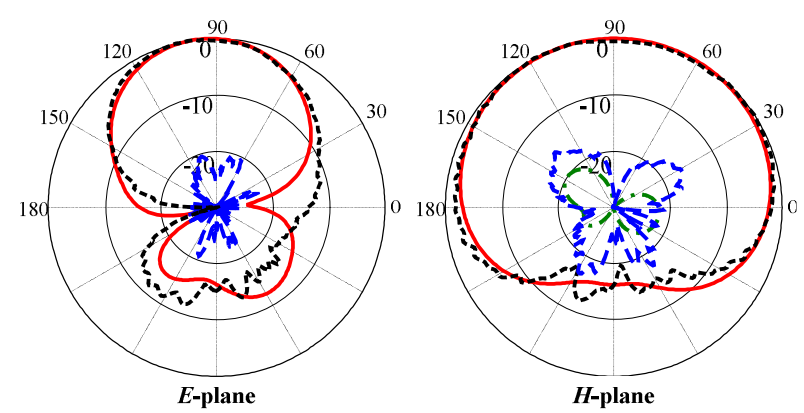

(b) $5.5 \mathrm{GHz}$

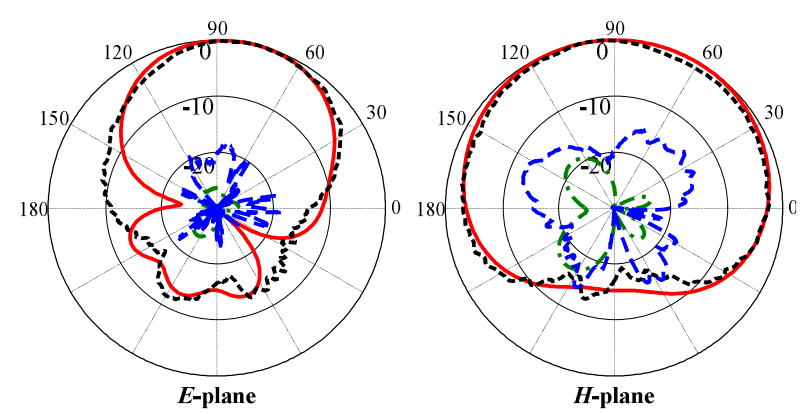

(c) $6 \mathrm{GHz}$

Fig. 9. Radiation patterns of the antenna. -....- mea. co-pol., - - - mea. x-pol., -... sim. x-pol.

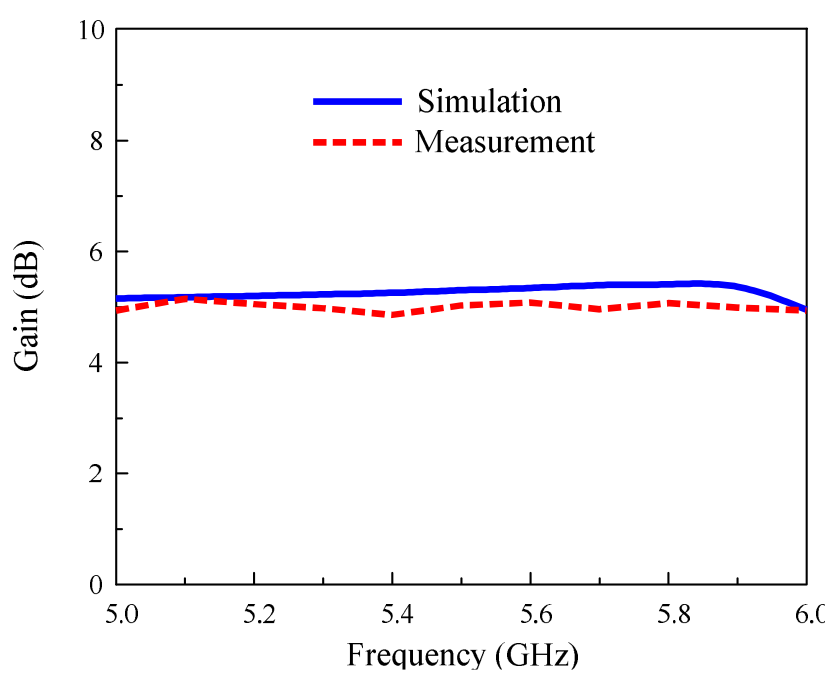

Fig. 10. Gain of the proposed antenna. 


\section{Conclusion}

A broadband quasi-Yagi antenna with a folded dipole driver was introduced. The measured bandwidth of the antenna was $4.67 \sim 6.26 \mathrm{GHz}$ for a $-10 \mathrm{~dB}$ reflection coefficient, and the measured gain was $4.86 \sim 5.15 \mathrm{dBi}$ within the bandwidth. With its broadband characteristics, planar structure, simple feed, stable radiation pattern, and small gain variation, this antenna is widely applicable to wireless communication systems, power combining, and phased arrays.

\section{References}

[1] Y. Qian, W. R. Deal, N. Kaneda, and T. Itoh, "Microstrip-fed quasi-Yagi antenna with broadband characteristics," Electron. Lett., vol. 34, no. 23, pp. 2194-2196, Nov. 1998.

[2] G. Zheng, A. A. Kishk, A. W. Glosson, and A. B. Yakovlev, "Simplified feed for modified printed Yagi antenna," Electron. Lett., vol. 40, no. 8, pp. 464-466, Apr. 2004.

[3] J. Sor, Y. Qian, and T. Itoh, "Coplanar waveguide fed quasi-Yagi antenna," Electron. Lett., vol. 36, no. 1, pp. 1-2, Jan. 2000.

[4] L. H. Truong, Y. H. Baek, M. K. Lee, S. W. Park, S. J. Lee, and J. K. Rhee, "A high-performance 94 $\mathrm{GHz}$ planar quasi-Yagi antenna on GaAs substrate," Micro. Opt. Techn. Lett., vol. 51, no. 10, pp. 23962400, Jul. 2009.

[5] H. K. Kan, R. B. Waterhouse, A. M. Abbosh, and M. E. Bialkowski, "Simple broadband planar CPWfed quasi-Yagi antenna," IEEE Antenna Wireless Propag. Lett., vol. 6, pp. 18-20, 2007.
[6] H. K. Kan, A. M. Abbosh, R. B. Waterhouse, and M. E. Bialkowski, "Compact broadband coplanar waveguide-fed curve quasi-Yagi antenna," IET Microw. Antennas Propag., vol. 1, no. 3, pp. 572-574, Jun. 2007.

[7] K. Han, Y. Park, and I. Park, "Broadband CPS-fed Yagi-Uda antenna," Electron. Lett., vol. 45, no. 24, pp. 1207-1209, Dec. 2009.

[8] S. X. Ta, B. Kim, H. Choo, and I. Park, "Slotline-fed quasi-Yagi antenna," The $9^{\text {th }}$ International Symposium on Antennas, Propagation, and EM Theory, ISAPE2010, 2010.

[9] P. R. Grajek, B. Shoenlinner, and G. M. Rebeiz, "A 24-GHz high-gain Yagi-Uda antenna array," IEEE Trans. Antennas Propag., vol. 52, no. 5 pp. 12571261, May 2004.

[10] E. Huang, T. Chiu, "Printed Yagi antenna with multiple reflectors," Electron. Lett., vol. 40, no. 19, pp. 1165-1166, Sep. 2004.

[11] E. Navarro, A. Martinez. J. Carrasco, and C. Reig, "A low-cost compact uniplanar quasi-Yagi printed antenna," Micro. Opt. Techn. Lett., vol. 50, no. 3, pp. 731-735, Mar. 2008.

[12] D. S. Woo, Y. G. Kim, K. W. Kim, and Y. K. Cho, "Design of quasi-Yagi antennas using an ultra-wideband balun," IEEE Micro. Opt. Techn. Lett., vol. 50, no. 8, pp. 2068-2071, Aug. 2008.

[13] R. Alhalabi, G. Rebeiz, "High-gain Yagi-Uda antennas for millimeter-wave switch-beam systems," IEEE Trans. Antenna Propag., vol. 57, no. 11, pp. 3672-3676, Nov. 2009.

[14] W. Tu, K. Chang, "Wide-band microstrip-to-coplanar stripline/slotline transition," IEEE Trans. Micro. Theo. Techn., vol. 54, no. 83, pp. 1084-1089, Mar. 2006. 
Son Xuat Ta

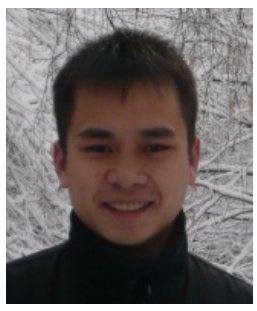

received the B.S. degree in Electronics and Telecommunications from Hanoi University of Science and Technology, Hanoi, Vietnam in 2008. He is currently studying a Ph.D. course in the School of Electrical and Computer Engineering, Ajou University, Suwon, Korea. His researches are focused on the widebands, multiband, and UWB antennas for next generation wireless communication systems.

\section{Byoungchul Kim}

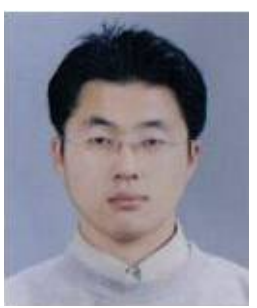

received the B.S. degree in Electronics Engineering from Dankook University, Korea in 2004, and the M.S. degree in Electrical and Computer Engineering from Ajou University, Suwon, Korea. He is currently studying a Ph.D. course in the School of Electrical and Computer Engineering, Ajou University, Korea. His researchs are focused on the antennas and RF devices.

\section{Hosung Choo}

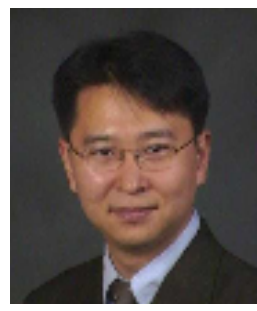

received the B.S. degree in Radiowave Engineering from Hanyang University, Korea in 1998, and the M.S. and Ph.D. degrees in Electrical Engineering from the University of Texas at Austin in 2000 and 2003, respectively. Since 2003, he has been a professor in the School of Electronic and Electrical Engineering at Hongik University, Seoul, Korea. His research interests include the small antenna, antenna design using optimization algorithms, tag and reader antenna for RFID.

\section{Ikmo Park}

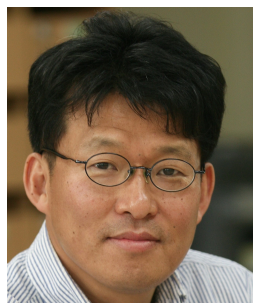

received the B.S. degree in Electrical Engineering from the State University of New York at Stony Brook in 1984, and the M.S. and Ph.D. degrees in Electrical Engineering from the University of Illinois at Urbana-Champaign in 1989 and 1994, respectively. From 1994 to 1996, he worked for LG Corporate Institute of Technology. Since 1996, he has been a professor in the School of Electrical and Computer Engineering at Ajou University, Suwon, Korea. His research interests include the design and analysis of terahetz and microwave passive devices and antennas. 